\title{
Assistência ao paciente com hipertensão na Atenção Primária à Saúde
}

\author{
Assistance to patients with hypertension in Primary Healty Care \\ Asistencia a pacientes con hipertensión en Atención Primaria
}

Recebido: 31/03/2021 | Revisado: 04/04/2021 |Aceito: 04/04/2021 | Publicado: 14/04/2021

Victor Guilherme Pereira da Silva Marques

ORCID: https://orcid.org/0000-0002-7441-9811

Centro Universitário do Piauí, Brasil

E-mail: guilhermevictor521@gmail.com

Mariel Wágner Holanda Lima

ORCID: https://orcid.org/0000-0001-5063-3891 Secretaria de educação do estado do Rio Grande do Norte, Brasil

E-mail: marielhoolanda@gmail.com

Ana Carla Almeida de Melo

ORCID: https://orcid.org/0000-0001-5853-8899 Universidade Potiguar, Brasil

E-mail: anacarla_melo@yahoo.com.br

Bruno Santos Souza

ORCID: https://orcid.org/0000-0001-5913-5258

Faculdade Ages de Medicina, Brasil

E-mail: enfobrunosouza@gmail.com

Bárbara Bispo de Santana

ORCID: https://orcid.org/0000-0002-7017-2401

Faculdade Ages de Jacobina, Brasil

E-mail: barbara_bispo12@hotmail.com

Rodrigo Andrade Leal

ORCID: https://orcid.org/0000-0002-4432-693X

Faculdade Ages de Medicina, Brasil

E-mail: rodrigo.enf2012@hotmail.com

Tarcísio Gonçalves de Souza Santos

ORCID: https://orcid.org/0000-0002-3748-9503

Faculdade Ages de Medicina, Brasil

E-mail: enfertarcisio@gmail.com

Marks Passos Santos

ORCID: https://orcid.org/0000-0003-1180-404X

Faculdade Ages de Medicina, Brasil

E-mail enfer.marks@hotmail.com

Lisandra Santana Oliveira Reis

ORCID: https://orcid.org/0000-0002-5249-5071

Universidade do Vale do Taquari, Brasil

E-mail: lisandrasoreis@outlook.com

Aranin Queiroz de Sousa Santos

ORCID: https://orcid.org/0000-0002-0195-1292

Faculdade Ages de Medicina, Brasil E-mail: enf.aranin@gmail.com

Leandro Luiz da Silva Loures

ORCID: https://orcid.org/0000-0001-8863-8858

Universidade Federal de Juiz de Fora, Brasil

E-mail: leandrolouresnutri@gmail.com

Célio Pereira de Sousa Júnior

ORCID: https://orcid.org/0000-0003-0726-0668 Universidade Federal do Pará, Brasil

E-mail: academicocelio@gmail.com

Emanuelly Marinho de Oliveira ORCID: https://orcid.org/0000-0002-1085-710X Universidade Pitágoras, Brasil

E-mail: emanuellymarinho21@gmail.com

Maria Sinária Silva de Castro dos Santos

ORCID: https://orcid.org/0000-0001-6659-4938 Universidade Pitágoras, Brasil E-mail: sinaryahc@gmail.com

Camila Almeida Bandeira

ORCID: https://orcid.org/0000-0002-5315-3313

Faculdade Internacional da Paraíba, Brasil

E-mail: camilabandeiranutri@hotmail.com 
Lorrane Silva de Souza

ORCID: https://orcid.org/0000-0002-0391-7952 Universidade Anhanguera, Brasil E-mail: lorrany.j95@hotmail.com

\begin{abstract}
Resumo
O presente estudo tem como objetivo realizar um levantamento bibliográfico acerca da assistência ao paciente com hipertensão na atenção primária à saúde. Trata-se de uma revisão integrativa da literatura, a busca ocorreu na Biblioteca Virtual em Saúde (BVS), com o auxílio das bases de dados Literatura Latino- Americana e do Caribe em Ciências da Saúde (LILACS), Scientific Electronic Library Online (SciELO), Base de Dados de Enfermagem (BDENF) e PUBMED. Os artigos foram coletados no período de janeiro a fevereiro de 2021. Foram tilizados os descritores: Atenção primária à saúde, Assistência ao paciente, Hipertensão e Promoção da saúde. Após a aplicação dos critérios de inclusão, restaram 20 estudos. O presente estudo demonstra que é necessário a atuação da equipe multidisciplinar em combate á hipertensão na atenção primária, utilizando de estratégias protocoladas pelo ministério da saúde se destacando a educação em saúde, que por meio das ações realizadas é possível manter o controle da doença em pacientes hipertensos.
\end{abstract}

Palavras-chave: Atenção primária à saúde; Assistência ao paciente; Hipertensão; Promoção da saúde.

\begin{abstract}
This study aims to conduct a literature survey about the care of patients with hypertension in primary health care. This is an integrative literature review, the search occurred in the Virtual Health Library (VHL), with the help of the databases Latin American and Caribbean Literature on Health Sciences (LILACS), Scientific Electronic Library Online (SciELO), Nursing Database (BDENF) and PUBMED. The articles were collected from January to February 2021. The following descriptors were used: Primary health care, Patient care, Hypertension, and Health promotion. After applying the inclusion criteria, 20 studies remained. This study shows that it is necessary the performance of the multidisciplinary team in combating hypertension in primary care, using the strategies protocoled by the Ministry of Health, highlighting health education, which through the actions taken is possible to maintain disease control in hypertensive patients.
\end{abstract}

Keywords: Primary health care; Patient care; Hypertension; Health promotion.

\title{
Resumen
}

Este estudio tiene como objetivo realizar un estudio bibliográfico sobre la atención a los pacientes con hipertensión en la atención primaria de salud. Se trata de una revisión bibliográfica integradora, la búsqueda ocurrió en la Biblioteca Virtual en Salud (BVS), con el auxilio de las bases de datos Latin American and Caribbean Literature on Health Sciences (LILACS), Scientific Electronic Library Online (SciELO), Nursing Database (BDENF) y PUBMED. Los artículos se recogieron de enero a febrero de 2021. Se utilizaron los siguientes descriptores: Atención primaria, Atención al paciente, Hipertensión y Promoción de la salud. Tras aplicar los criterios de inclusión, quedaron 20 estudios. Este estudio demuestra que es necesaria la actuación del equipo multidisciplinar en la lucha contra la hipertensión en atención primaria, utilizando las estrategias protocolizadas por el ministerio de sanidad destacando la educación sanitaria, que a través de las acciones realizadas es posible mantener el control de la enfermedad en los pacientes hipertensos.

Palabras clave: Atención primaria; Atención al paciente; Hipertensión; Promoción de la salud.

\section{Introdução}

A Atenção Primária à Saúde (APS) configura-se como o contato preferencial do usuário com o sistema de saúde e o local responsável pela organização do cuidado à sua saúde, de suas famílias e da população. Por isso deve ser orientada pelos princípios do primeiro contato, longitudinalidade, integralidade, coordenação, abordagem familiar e enfoque comunitário (Dantas \& Roncalli, 2019).

A hipertensão arterial sistêmica (HAS) representa um sério problema epidemiológico no Brasil, tanto pela sua elevada prevalência na população adulta e idosa, quanto pelas complicações que acarreta, com acentuadas taxas de morbimortalidade e impactos relevantes nos custos hospitalares, previdenciários, econômicos e sociais. É uma síndrome de origem multifatorial caracterizada pelo aumento das cifras pressóricas arteriais, possibilitando anormalidades cardiovasculares e metabólicas, que podem levar a alterações funcionais e/ou estruturais de vários órgãos, principalmente coração, cérebro, rins e vasos periféricos (Camargo, Anjos \& Amaral, 2013). 
A hipertensão arterial possui elevados custos sociais, porque se tem de enfrentar dois grandes desafios: o primeiro é a adesão do paciente ao tratamento; o segundo se refere à colocação dos pacientes que aderem ao tratamento dentro das metas preconizadas pelas diversas diretrizes publicadas pelas sociedades científicas nacionais e internacionais. Além disso, por ser uma doença crônica, requer, geralmente, o tratamento com medicamento de uso contínuo, sendo comum a associação de outros medicamentos para o tratamento de doenças associadas ou de complicações do próprio quadro hipertensivo. Isto aumenta a possibilidade de ocorrência de interações medicamentosas e de Problemas Relacionados aos Medicamentos (PRM). Seu tratamento requer a ação de uma equipe multiprofissional para que se alcance às metas esperadas e o controle do paciente hipertenso (Martins et al., 2014).

A idade avançada configura-se o principal fator de risco da HAS, principalmente indivíduos a partir dos 50 anos de idade, havendo maior prevalência entre os indivíduos do gênero masculino, porém com prevalência parecida entre ambos. Outros fatores de risco aparecem o excesso de peso, sedentarismo, ingesta aumentada de sal nas refeições, uso de álcool, além dos fatores socioeconômicos e genéticos (Carvalho et al., 2013).

Evidenciam-se pela equipe que atua na Estratégia de Saúde da Família imensas dificuldades, dentre estas o cuidado às pessoas com Hipertensão Arterial Sistêmica (HAS). No cuidado à pessoa com HAS, é importante que a equipe da ESF participe de processos de construção de conhecimento, assimilando e utilizando inovações, tanto tecnológicas como humanísticas, de forma equilibrada, para que se possa oferecer condições a essas pessoas de desenvolver o autocuidado de maneira mais adequada ao seu contexto sociocultural (Silva et al., 2018).

O controle dessas condições nos indivíduos afetados - isto é, a manutenção dos níveis tensionais ou glicêmicos em determinados limites de normalidade - depende de uma série de fatores, desde a adoção de estilos de vida que envolva atividade física e dieta adequada ao uso continuado de medicamentos, sendo assim fortemente determinado pelas condições de vida e acesso a serviços de saúde de qualidade (Bortoluz, Lima \& Nedel, 2016).

O HiperDia constitui um sistema de cadastramento e acompanhamento de hipertensos e diabéticos, em que os profissionais de saúde são responsáveis pelo atendimento aos usuários e preenchimento de dados. Visa ao monitoramento dos pacientes captados no Plano Nacional de Reorientação da Atenção à HAS e ao DM (Diabetes Mellitus) e à geração de informação para aquisição, dispensação e distribuição de medicamentos regular e continuamente (Filha, Nogueira \& Medina, 2014)

Um dos maiores desafios no combate à hipertensão arterial ainda se deve à não adesão ao tratamento. Estudos mostram baixos níveis de adesão à terapia anti-hipertensiva, além de os maiores índices estarem associados a serviços de saúde especializados. Outras formas de manejo da hipertensão arterial, como a prática de atividade física e a dieta também são importantes. Alguns estudos mostram baixa adesão a essas práticas na população em geral, e em grupos específicos, como os hipertensos e diabéticos, mais sujeitos aos efeitos danosos do sedentarismo e da dieta não adequada (Girotto, Andrade, Cabrera \& Matsuo, 2013).

É válido considerar a prevalência da não adesão ao tratamento como indicador de problemas na qualidade do processo de cuidado em saúde. Além disso, a adesão ao tratamento é essencial na assistência de enfermagem ao indivíduo hipertenso, pois com ela o profissional poderá desenvolver intervenções clínicas e educativas que se ajustem às reais necessidades dos usuários e às de grupos que tenham as mesmas dificuldades em aderir (Reiners et al., 2012).

Para poderem atuar de maneira eficaz, propondo e implementando ações que atendam às reais necessidades desta população, os profissionais precisam conhecer os pacientes e identificar os que aderem e os que não aderem ao tratamento, bem como levantar os motivos pelos quais os hipertensos assistidos não estão seguindo o tratamento (Reiners et al., 2012).

O presente estudo tem como objetivo realizar um levantamento bibliográfico acerca da assistência ao paciente com hipertensão na atenção primária à saúde. 


\section{Metodologia}

Trata-se de um estudo descritivo, do tipo revisão integrativa de literatura, de caráter qualitativo uma vez que é definida como um tipo de investigação voltada para o aspecto qualitativo de uma determinada questão, nesse caso, a importância da assistência ao paciente com hipertensão na atenção primária à saúde. A revisão de literatura permite aprofundar dentro de diversos autores e referenciais, sobre os discursos e principais temas abordados (Pereira et al., 2018).

Para direcionar a presente revisão delineou-se como questão norteadora: "Qual a importância da assistência ao paciente com hipertensão na atenção primária à saúde?".

Para a construção deste trabalho, a busca dos artigos foi realizada na Biblioteca Virtual em Saúde (BVS), com o auxílio das bases de dados Literatura Latino-Americana e do Caribe em Ciências da Saúde (LILACS), Scientific Electronic Library Online (SciELO), Base de Dados de Enfermagem (BDENF) e PUBMED. Os artigos foram coletados no período de janeiro a fevereiro de 2021. Foram utilizados os descritores: "Atenção primária à saúde", "Assistência ao paciente", "Hipertensão" e "Promoção da saúde", localizados na lista dos Descritores em Ciências da Saúde, disponível no Portal da Biblioteca Virtual em Saúde (http://decs.bvs.br).

Foram selecionados como critérios de inclusão: artigos completos disponíveis integralmente nas bases de dados elencadas, em idiomas português, espanhol e inglês, com o recorte temporal de 2011 a 2021 e relacionados com a temática. Foram excluídos artigos duplicados, incompletos, resumos, resenhas, debates, artigos publicados em anais de eventos e indisponíveis na íntegra.

\section{Resultados e Discussão}

A partir da revisão de literatura e análise dos estudos indexados nas bases de dados eletrônicas, acerca da temática proposta, foram encontrados 150 estudos científicos, sendo que, apenas 80 estudos foram selecionados, 50 atenderam aos critérios de inclusão previamente estabelecidos, destes, 30 foram excluídos com base nos critérios de exclusão, restando 20 artigos para composição e análise do estudo. O fluxograma com o detalhamento das etapas de pesquisa está apresentado a seguir na Figura 1. 
Figura 1. Fluxograma de identificação e seleção dos artigos.2021.

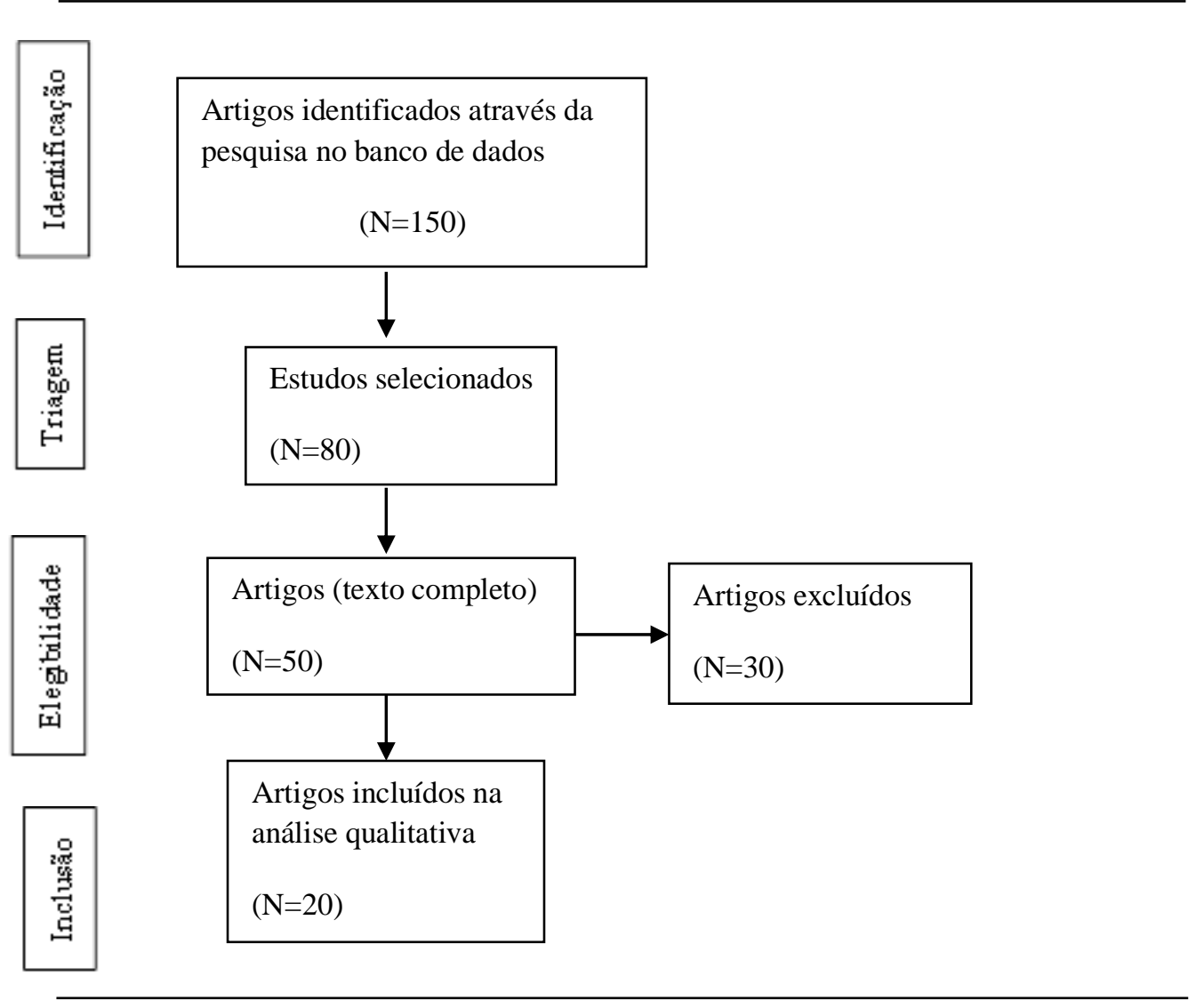

Fonte: Autores (2021).

Para análise, interpretação, discussão e elaboração dos dados, foram explorados 20 estudos compreendidos entre os anos 2011 a 2021. Apresentando os fatores que levam a assistência ao paciente com hipertensão na atenção primária à saúde.

Entende-se que o trabalho em equipe multiprofissional e interdisciplinar na Estratégia Saúde da Família (ESF) aos portadores de HAS visa à efetivação da integralidade em saúde no sentido de possibilitar uma atuação em saúde mais contextualizada, resolutiva na perspectiva da corresponsabilização de trabalhadores, usuários e suas famílias. As ações devem ser comuns a todos no planejamento e na promoção à saúde (ações educativas em relação à mudança do estilo de vida, fatores de risco e produção de material educativo), no treinamento de profissionais, no encaminhamento a outros profissionais, quando for necessário, nas ações assistenciais individuais e em grupo, na participação em projeto de pesquisa e no gerenciamento do programa. A ESF propõe-se as superar práticas estritamente curativas, fragmentadas, em que predominam a preocupação e a realização de procedimentos, utilização de equipamentos e reprodução de normas preestabelecidas, como o cuidado em si, que muitas vezes não atendem às necessidades de saúde das famílias e usuários (Camargo, Anjos \& Amaral, 2013).

O acesso garantido aos serviços de saúde pública se faz essencial para o usuário hipertenso. Especialmente aquele em situação de alto risco cardiovascular deve ser assistido na APS pelas equipes de saúde da família, visando à adesão ao tratamento; ao autocuidado, e à responsabilidade compartilhada. Entretanto, embora a Estratégia de Saúde da Família seja cenário privilegiado para intervenções de base comunitária direcionadas à prevenção integrada dos fatores de risco cardiovascular, há fragilidades no manejo do portador de HAS (Nobre et al.,2020).

Todavia, ressalta-se que o comparecimento às consultas pelos idosos de maneira regular leva o indivíduo ao acompanhamento adequado da HAS, tornando-se um fator positivo na adesão ao tratamento pelo monitoramento durante as 
consultas, com a finalidade de esclarecimento sobre a importância deste acompanhamento, e pela apresentação de melhores medidas a serem seguidas pelos idosos (Ferreira et al., 2019).

O acompanhamento dos hipertensos pressupõe boa adesão ao serviço e, consequentemente, o controle adequado dos níveis pressóricos. Porém, uma boa adesão consiste em uma atitude global em relação à própria saúde e exige participação ativa dos hipertensos, não como um objeto, mas como sujeito do processo. Para isso é necessário o comparecimento às consultas e a mensuração regular da pressão arterial a fim de avaliar o controle da hipertensão (Silva et al., 2013).

No que se refere ao vínculo, a integralidade e a satisfação das necessidades são produtos do processo de trabalho dos profissionais que prestam a assistência de saúde. Durante o atendimento, é necessário que o princípio da integralidade seja respeitado. Para isso, faz-se necessário acolhimento, escuta, ações resolutivas e práticas humanizadas promotoras da empatia a entre o usuário e o profissional de saúde, de modo a facilitar a adesão ao projeto terapêutico e o sucesso do tratamento. Estudos destacam que os usuários valorizam a atenção do profissional de saúde durante o atendimento, desde a recepção até o momento da consulta. A formação do vínculo entre a ESF e o usuário garante laços de confiança e corresponsabilidade entre profissionais e usuários (Silva et al., 2013).

A verificação da pressão arterial é fundamental no momento da consulta de enfermagem. Deve ser realizada em toda consulta após deixar o paciente em repouso por pelo menos 5 minutos em ambiente calmo ao chegar à unidade de saúde. Vale ressaltar que o enfermeiro deve se certificar que o paciente não está com a bexiga cheia e utilizar manguitos de dimensões recomendadas para garantir a acurácia das medidas de pressão arterial (Branco et al., 2013).

As consultas médicas e de enfermagem para acompanhamento dos casos que atingiram a meta pressórica deverão ser preferencialmente intercaladas. Sugere-se que sua periodicidade varie de acordo com o risco cardiovascular estabelecido por meio do escore de Framingham e de acordo com as necessidades individuais, considerando-se as diretrizes locais (Brasil, 2014).

Caso a PA não diminua com o uso da medicação indicada até a segunda semana de tratamento, após certificar-se que o paciente está fazendo uso correto da medicação prescrita, a equipe de Enfermagem deverá orientar o paciente para consultar com seu médico. Se o paciente não estiver usando corretamente a medicação, a equipe de Enfermagem deverá refazer a orientação sobre o uso da medicação e continuar monitorando a PA (Brasil, 2014).

A adesão ao tratamento farmacológico envolve diferentes elementos que constituem esse processo: o indivíduo, o tratamento, a doença, os serviços, os profissionais de saúde, bem como o meio social e cultural do usuário e de sua família. Para que a adesão seja alcançada, são necessários o alinhamento e a organização desses elementos (Gewehr, Gelatti, Colet \& Oliveira, 2018).

Cabe enfatizar que, na avaliação da adesão do paciente ao tratamento medicamentoso prescrito, devem-se considerar os fatores externos ao paciente, mas também sua compreensão do tratamento e a relação estabelecida com os profissionais de saúde. A comunicação entre os profissionais de saúde bem como entre profissionais e pacientes é extremamente importante, uma vez que a falta de conhecimento ou a existência de dúvida por parte do paciente constituem, também, fatores de nãoadesão (Ferreira et al.,2013).

A adesão terapêutica é a extensão em que a pessoa segue as recomendações do profissional de saúde relacionadas à adoção do uso de medicamentos e mudanças no estilo de vida. Destarte, é relevante entender o comportamento dos pacientes que não aderem ao tratamento, para que os profissionais de saúde envolvidos no atendimento, principalmente o enfermeiro, compreendam sua realidade para poderem agir comunicativamente com eles, dando-lhes orientações para motivar a adesão ao tratamento e, ao mesmo tempo, perceberem-se como agentes de mudança, a partir da implementação de programas educativos e avaliativos voltados à melhoria das ações desenvolvidas, buscando a adaptação destas atividades à realidade dos indivíduos e assim contribuindo para melhorar e manter sua saúde (Waidman et al., 2012). 
A educação em saúde pode ser uma ferramenta útil para minimizar ou evitar o impacto desses fatores sobre a adesão aos fármacos para HAS. Assim, torna-se relevante que o enfermeiro realize ações de acompanhamento e monitoramento da saúde desses idosos, visando contribuir na identificação de dificuldades acerca da adesão, bem como no fornecimento de orientações (Tavares et al., 2016).

A maior utilização de ações educativas referentes à motivação e ao direcionamento ao autocuidado, além do estabelecimento de vínculos na relação paciente/ profissional de saúde, como base de sustentação para a implementação de uma abordagem multidisciplinar e individualizada de atenção à saúde, pode contribuir com o processo de adesão terapêutica medicamentosa em indivíduos portadores de HA. Para completar, a orientação e a escolha de drogas com menos efeitos indesejáveis, baixo custo, monoterapia, comodidade posológica, combinação terapêutica adequada, prescrição e informações por escrito para fácil entendimento, familiarização dos médicos com esquemas terapêuticos e tratamento para grupos diferenciados podem contribuir para o seguimento correto dos medicamentos (Daniel \& Veiga, 2013).

\section{Conclusão}

O presente estudo demonstra que é necessário a atuação da equipe multidisciplinar em combate á hipertensão na atenção primária, utilizando de estratégias protocoladas pelo ministério da saúde se destacando a educação em saúde, que por meio das ações realizadas é possível manter o controle da doença em pacientes hipertensos.

A adesão ao tratamento farmacológico se dá por diversos fatores incluindo a vontade do paciente de aderir o tratamento de forma correta e as ações realizadas pelos profissionais da saúde, seguindo os protocolos existentes e buscando a redução de agravamento da hipertensão nesses pacientes que estão sendo rastreados pela equipe da Estratégia Saúde da Família.

Conclui-se com esse estudo de revisão integrativa, é relevante para a literatura no tocante ao incentivo do conhecimento teórico-prático acerca da assistência ao paciente com hipertensão na atenção primária à saúde.

Neste sentido, essa pesquisa contribui não somente para a formação acadêmica, mas sugere e estimula a produção de novas pesquisas de temática similar que abordem a importância da assistência ao paciente com hipertensão na atenção primária à saúde para que se possa contribuir futuramente na melhor qualidade de vida da sociedade em geral.

\section{Referências}

Bortoluz, S., de Lima, L. A., \& Nedel, F. B. (2016). Condições de saúde e utilização de um serviço de atenção primária em pacientes hipertensos e/ou diabéticos. Ciência \& Saúde, 9(3), 156-166.

Branco, C. S. N., Mendes, R. S., de Oliveira, S. K. P., \& Pamplona, Y. D. A. P. (2013). Consulta de enfermagem ao paciente com hipertensão na estratégia de saúde da família. Revista Enfermagem Contemporânea, 2(2), 196-208.

Brasil. (2014). Estratégias para o cuidado da pessoa com doença crônica: hipertensão arterial sistêmica / Ministério da Saúde, Secretaria de Atenção à Saúde, Departamento de Atenção Básica - Brasília: Ministério da Saúde.

Camargo, R. A. A., Anjos, F. R., \& Amaral M. F. (2013). Estratégia saúde da família nas ações primárias de saúde ao portador de hipertensão arterial sistêmica. Rev Min Enf, 17(4), 864-872.

Carvalho, M. V. D., Siqueira, L. B., Sousa, A. L. L., \& Jardim, P. C. B. V. (2013). A influência da hipertensão arterial na qualidade de vida. Arquivos Brasileiros de Cardiologia, 100(2), 164-174.

Daniel, A. C. Q. G., \& Veiga, E. V. (2013). Fatores que interferem na adesão terapêutica medicamentosa em hipertensos. Einstein (São Paulo), 11(3), 331337.

Dantas, R. C. D. O., \& Roncalli, A. G. (2019). Protocolo para indivíduos hipertensos assistidos na Atenção Básica em Saúde. Ciência \& Saúde Coletiva, 24(1), 295-306.

Ferreira, F. M., Cruz, M. J. B., Santos, D. F., Linhares, M. P., \& Andrade, R. A. (2013). Fatores relacionados à adesão farmacoterapêutica de pacientes hipertensos acolhidos na estratégia de saúde da família. Revista de APS, 16(3), 258-268. 
Research, Society and Development, v. 10, n. 4, e36010414523, 2021 (CC BY 4.0) | ISSN 2525-3409 | DOI: http://dx.doi.org/10.33448/rsd-v10i4.14523

Filha, F. S. S.C., Nogueira, L. T., \& Medina, M. G. (2014). Avaliação do controle de hipertensão e diabetes na Atenção Básica: perspectiva de profissionais e usuários. Saúde em Debate, 38, 265-278.

Ferreira, E. A., Alves, D. C. S. Q., Parnaíba, F. J. B., de Araújo, R. V., Vieira, G. P., de Alencar, A. P., \& Amorim, R. M. L. (2019). Vínculo ProfissionalUsuário na Estratégia Saúde da Família: Percepções de Idosos Hipertensos. ID on line REVISTA DE PSICOLOGIA, 13(43), 748-760.

Gewehr, D. M., Bandeira, V. A. C., Gelatti, G. T., Colet, C. D. F., \& Oliveira, K. R. D. (2018). Adesão ao tratamento farmacológico da hipertensão arterial na Atenção Primária à Saúde. Saúde em Debate, 42(116), 179-190.

Girotto, E., Andrade, S. M. D., Cabrera, M. A. S., \& Matsuo, T. (2013). Adesão ao tratamento farmacológico e não farmacológico e fatores associados na atenção primária da hipertensão arterial. Ciência \& Saúde Coletiva, 18(6), 1763-1772.

Martins, B. C. C., Firmino, P. Y. M., Alves, S. C. F., Lima, L. F., Oliveira, B. E., Porto, A. C., Vieira, V. M. S. F., Mota, M. V., Ponciano, A. M. S., Fonteles, M. M. F. (2014). Adesão ao tratamento para hipertensão em uma Unidade Básica de Saúde do Ceará. Rev Bras Hipertens, 21(1), 24-30.

Nobre, A. L. C. S. D., Lima, C. D. A., Oliveira, M. J. L. D., Vieira, D. D. M. A., Martelli Júnior, H., \& Costa, S. D. M. (2020). Hipertensos assistidos em serviço de atenção secundária: risco cardiovascular e determinantes sociais de saúde. Cadernos Saúde Coletiva, 28(3), 334-344.

Pereira, A. S., et al. (2018). Metodologia da pesquisa científica. UFSM. https://repositorio.ufsm.br/bitstream/handle/1/15824/Lic_Computacao_MetodologiaPesquisa-Cientifica.pdf? sequence $=1$.

Reiners, A. A. O., Seabra, F. M. F., de Souza Azevedo, R. C., Sudré, M. R. S., \& Duarte, S. J. H. (2012). Adesão ao tratamento de hipertensos da atenção básica. Ciência, Cuidado e Saúde, 11(3), 581-587.

Silva, C. S., Paes, N. A., Figueiredo, T. M. R. M. D., Cardoso, M. A. A., Silva, A. T. M. C. D., \& Araújo, J. S. S. D. (2013). Controle pressórico e adesão/vínculo em hipertensos usuários da Atenção Primária à Saúde. Revista da Escola de Enfermagem da USP, 47(3), 584-590.

Silva, P. C. D. S. D., Negrão, M. D. L. B., Gimenes, F. R. E., Chini, L. T., Gonçalves, M. F. C., \& Fava, S. M. C. L. (2018). Ressignificação do cuidado às pessoas com hipertensão arterial sistêmica. Rev. enferm. UFPE on line, 12(7), 1940-1948.

Tavares, D. M. D. S., Guimarães, M. D. O., Ferreira, P. C. D. S., Dias, F. A., Martins, N. P. F., \& Rodrigues, L. R. (2016). Qualidade de vida e adesão ao tratamento farmacológico entre idosos hipertensos. Revista Brasileira de Enfermagem, 69(1), 134-141.

Waidman, M. A. P., Radovanovic, C. A. T., Estevam, M. C., \& Marcon, S. S. (2012). Assistência à pessoa com hipertensão arterial na ótica do profissional de saúde. Revista Brasileira de Enfermagem, 65(3), 445-453. 